\title{
Three-Family Supersymmetric Standardlike Models from Intersecting Brane Worlds
}

\author{
Mirjam Cvetič, ${ }^{1,2}$ Gary Shiu, ${ }^{1,2}$ and Angel M. Uranga ${ }^{2}$ \\ ${ }^{1}$ Department of Physics and Astronomy, University of Pennsylvania, Philadelphia, Pennsylvania 19104-6396 \\ ${ }^{2}$ Theory Division, CERN, CH-1211 Geneva 23, Switzerland
}

(Received 20 July 2001; published 24 October 2001)

\begin{abstract}
We construct the first three family $N=1$ supersymmetric string model with standard model gauge group $\mathrm{SU}(3)_{C} \times \mathrm{SU}(2)_{L} \times \mathrm{U}(1)_{Y}$ from an orientifold of type IIA theory on $\mathbf{T}^{6} /\left(\mathbf{Z}_{2} \times \mathbf{Z}_{2}\right)$ and D6branes intersecting at angles. In addition to the minimal supersymmetric standard model particles, the model contains right-handed neutrinos, a chiral (but anomaly-free) set of exotic multiplets, and extra vectorlike multiplets. We discuss some phenomenological features of this model.
\end{abstract}

DOI: $10.1103 /$ PhysRevLett.87.201801

The space of classical string vacua is highly degenerate, and at present we are unable to make definitive statements about how the string vacuum describing our universe is selected. Nonetheless, one can use phenomenological constraints as guidelines to construct semirealistic string models and explore, with judicious assumptions, the resulting phenomenology. The purpose of such explorations is, of course, not to find the model which would fully describe our world, but to examine the generic features of these string derived solutions.

Until a few years ago, such explorations were carried out mainly in the framework of weakly coupled heterotic string theory. Indeed, a number of semirealistic string models have been constructed and analyzed [1]. However, an important lesson from string duality is that these models represent only a corner of $M$ theory - the string vacuum describing our world may well be in a completely different regime in which the perturbative description of heterotic string theory breaks down [2]. Fortunately, the advent of D-branes allows for the construction of semirealistic string models in another calculable regime, as illustrated by the various four-dimensional $N=1$ supersymmetric type II orientifolds ([3-12] and references therein) constructed using conformal field theory techniques. However, the constraints on supersymmetric fourdimensional models are rather restrictive, leading to not fully realistic gauge sectors and matter contents. Motivated by the search for standard model-like solutions, several discrete or continuous deformations of this class of models have been explored. They include the following: (i) blowing-up of orientifold singularities $[13,14]$, (ii) locating the branes at different points in the internal space (see, e.g., $[9,12,15])$ which in a $T$-dual picture corresponds to turning on continuous or discrete Wilson lines, (iii) introduction of discrete values for the Neveu-Schwarz-Neveu-Schwarz (NS-NS) $B$ field $[7,16]$ which in the $T$-dual picture corresponds to tilting the compactification tori, (iv) introduction of gauge fluxes in the D-brane world volumes (see [17] for an earlier discussion, and $[18,19]$ for supersymmetric $D=6$ models), which in the $T$-dual version corresponds to D-branes intersecting at angles (hence closely related to models in [20,21]).
PACS numbers: $11.10 . \mathrm{Kk}, 11.25 . \mathrm{Mj}, 12.60 . \mathrm{Jv}, 98.80 . \mathrm{Cq}$

An appealing feature of (iv) is that generically, there exists chiral fermions where D-branes intersect [22]. Their multiplicity is hence determined by a topological quantity, i.e., the intersection number of the branes. Models with D-branes intersecting at arbitrary angles are nonsupersymmetric. For nonsupersymmetric models, Ramond-Ramond (RR) tadpole cancellation conditions are less constraining [23], a fact exploited to construct semirealistic models in $[24,25]$, and more recently in $[19,21,26-28]$ in the context of intersecting branes. However, nonsupersymmetric models suffer from a less understood, complicated dynamics. At the quantum level, flat directions are lifted, leading to involved stabilization problems. In addition, uncanceled NS-NS tadpoles require redefining the background geometry [29]. These difficulties are the main reasons that we focus on string models with $N=1$ supersymmetry.

The purpose of this Letter is to present the first example of a four-dimensional $N=1$ supersymmetric type IIA orientifold with D6-branes intersecting at angles, leading to standard model gauge group (as part of the gauge group structure) and three quark-lepton families. Beyond the structure of the minimal supersymmetric standard model (MSSM), the model contains some additional gauge factors, right-handed neutrinos, a chiral set of fields with exotic standard model gauge quantum numbers, and diverse vectorlike multiplets. Despite its lack of fully realistic features, it provides the first construction of supersymmetric standard model-like string models in the setup of (nontrivial) intersecting brane worlds. Interestingly, since only D6-branes and O6-planes are involved, the $M$ theory lift of this general class of supersymmetric orientifold models corresponds to purely geometrical backgrounds admitting a $G_{2}$ holonomy metric and leading to chiral fourdimensional fermions.

We shall provide the key features of the construction, the gauge group structure, and the massless spectrum. The details of the construction, consistency conditions, as well as a broader class of models (including examples of grand unified theories) will be presented in a companion paper [30].

The construction of the model is based on an orientifold of type IIA on $\mathbf{T}^{6} /\left(\mathbf{Z}_{2} \times \mathbf{Z}_{2}\right)$ (related to [4] by $T$ duality), 
with D6-branes not parallel to the orientifold 6-planes (O6-planes). The generators $\theta, \omega$ act as $\theta:\left(z_{1}, z_{2}, z_{3}\right) \rightarrow$ $\left(-z_{1},-z_{2}, z_{3}\right)$, and $\omega:\left(z_{1}, z_{2}, z_{3}\right) \rightarrow\left(z_{1},-z_{2},-z_{3}\right)$ on the complex coordinates $z_{i}$ of $\mathbf{T}^{6}$, which we moreover choose to be factorizable. The orientifold action is $\Omega R$, where $\Omega$ is world-sheet parity, and $R$ acts by $R:\left(z_{1}, z_{2}, z_{3}\right) \rightarrow$ $\left.\bar{z}_{1}, \bar{z}_{2}, \bar{z}_{3}\right)$. The model contains four kinds of O6-planes, associated with the actions of $\Omega R, \Omega R \theta, \Omega R \omega, \Omega R \theta \omega$. We will focus on the open string (charged) spectrum. The closed string sector contains gravitational supermultiplets as well as orbifold moduli and is straightforward to determine. The cancellation of the RR cross-cap tadpoles requires an introduction of $K$ stacks of $N_{a}$ D6-branes $(a=1, \ldots, K)$ wrapped on three cycles [taken to be the product of 1-cycles $\left(n_{a}^{i}, m_{a}^{i}\right)$ in the $i$ th two-torus], and their images under $\Omega R$, wrapped on cycles $\left(n_{a}^{i},-m_{a}^{i}\right)$.

The rules to compute the spectrum are analogous to those in [19]. Consequently, models with all tori orthogonal lead to an even number of families. Hence, we consider models with one tilted $T^{2}$, where the tilting parameter is discrete and has a unique nontrivial value [31]. This mildly modifies the closed string sector, but has an important impact on the open string sector. Namely, a D-brane 1-cycle $\left(n_{a}^{i}, m_{a}^{i}\right)$ along a tilted torus is mapped to $\left(n^{i},-m^{i}-n^{i}\right)$. It is convenient to define $\tilde{m}^{i}=m^{i}+\frac{1}{2} n^{i}$, and label the cycles as $\left(n^{i}, \tilde{m}^{i}\right)$.

The orbifold actions on the Chan-Paton indices of the branes, for each stack of $\mathrm{D}_{a}$-branes, and their $\Omega R$ images, denoted by $\mathrm{D}_{a^{\prime}}$-branes, are as follows:

$$
\begin{aligned}
& \gamma_{\theta, a}=\operatorname{diag}\left(i \mathbf{1}_{N_{a} / 2},-i \mathbf{1}_{N_{a} / 2} ;-i \mathbf{1}_{N_{a} / 2}, i \mathbf{1}_{N_{a} / 2}\right) \text {, } \\
& \gamma_{\omega, a}=\operatorname{diag}\left[\left(\begin{array}{cc}
0 & \mathbf{1}_{N_{a} / 2} \\
-\mathbf{1}_{N_{a} / 2} & 0
\end{array}\right) ;\left(\begin{array}{cc}
0 & \mathbf{1}_{N_{a} / 2} \\
-\mathbf{1}_{N_{a} / 2} & 0
\end{array}\right)\right] \text {, } \\
& \gamma_{\Omega R, a}=\left(\begin{array}{cccc} 
& & \mathbf{1}_{N_{a} / 2} & 0 \\
& & 0 & \mathbf{1}_{N_{a} / 2} \\
\mathbf{1}_{N_{a} / 2} & 0 & & \\
0 & \mathbf{1}_{N_{a} / 2} & &
\end{array}\right) .
\end{aligned}
$$

The model is constrained by RR tadpole cancellation conditions. In $\Omega R$ orientifolds twisted tadpoles vanish automatically [20,21], whereas untwisted RR tadpoles require cancellation of D6-brane and O6-plane 7-form charges. For models with a tilted third two-torus,

$$
\begin{gathered}
\sum_{a} N_{a} n_{a}^{1} n_{a}^{2} n_{a}^{3}-16=0, \\
\sum_{a} N_{a} n_{a}^{1} m_{a}^{2} \tilde{m}_{a}^{3}+8=0, \\
\sum_{a} N_{a} m_{a}^{1} n_{a}^{2} \tilde{m}_{a}^{3}+8=0, \\
\sum_{a} N_{a} m_{a}^{1} m_{a}^{2} n_{a}^{3}+16=0 .
\end{gathered}
$$

The solutions of the above equations define a consistent model; the construction of the resulting spectrum is discussed in detail in [30]. Here we summarize only the results for D6-branes not parallel to O6-planes (for zero angles, the spectrum follows from [4]). The $a a$ sector (strings stretched within a single stack of $\mathrm{D6}_{a}$-branes) is invariant under $\theta, \omega$, and is exchanged with $a^{\prime} a^{\prime}$ by the action of $\Omega R$. For the gauge group, the $\theta$ projection breaks $U\left(N_{a}\right)$ to $U\left(N_{a} / 2\right) \times U\left(N_{a} / 2\right)$, and $\omega$ identifies both factors, leaving $U\left(N_{a} / 2\right)$. Concerning the matter multiplets, we obtain three adjoint $N=1$ chiral multiplets.

The $a b+b a$ sector, strings stretched between $\mathrm{D}_{a}$ - and $\mathrm{D}_{b}$-branes, is invariant, as a whole, under the orbifold projections, and is mapped to the $b^{\prime} a^{\prime}+a^{\prime} b^{\prime}$ sector by $\Omega R$. The matter content before any projection would be given by $I_{a b}$ chiral fermions in the bifundamental $\left(N_{a}, \bar{N}_{b}\right)$ of $U\left(N_{a}\right) \times U\left(N_{b}\right)$, where
$I_{a b}=\left(n_{a}^{1} m_{b}^{1}-n_{b}^{1} m_{a}^{1}\right)\left(n_{a}^{2} m_{b}^{2}-n_{b}^{2} m_{a}^{2}\right)\left(n_{a}^{3} \tilde{m}_{b}^{3}-n_{b}^{3} \tilde{m}_{a}^{3}\right)$

is the intersection number of the wrapped cycles, and the sign of $I_{a b}$ denotes the chirality of the corresponding fermion $(I<0$ giving left-handed fermions in our convention). For supersymmetric intersections, additional massless scalars complete the corresponding chiral supermultiplet. In principle, one needs to take into account the orbifold action on the intersection point. However, the final result turns out to be insensitive to this subtlety and is still given by $I_{a b}$ chiral multiplets in the $\left(N_{a} / 2, \overline{N_{b} / 2}\right)$ of $U\left(N_{a} / 2\right) \times U\left(N_{b} / 2\right)$. A similar effect takes place in $a b^{\prime}+b^{\prime} a$ sector, for $a \neq b$, where the final matter content is given by $I_{a b^{\prime}}$ chiral multiplets in the bifundamental $\left(N_{a} / 2, N_{b} / 2\right)$.

For the $a a^{\prime}+a^{\prime} a$ sector the orbifold action on the intersection points turns out to be crucial. For intersection points invariant under the orbifold, the orientifold projection leads to a two-index antisymmetric representation of $U\left(N_{a} / 2\right)$, except for states with $\theta$ and $\omega$ eigenvalue +1 , where it yields a two-index symmetric representation. For points not fixed under some orbifold element, say two points fixed under $\omega$ and exchanged by $\theta$, one simply keeps one point, and does not impose the $\omega$ projection. Equivalently, one considers all possible eigenvalues for $\omega$, and applies the above rule to read off whether the symmetric or the antisymmetric survives. A closed formula for the chiral piece in this sector is given in [30].

The condition that the system of branes preserve the $N=1$ supersymmetry requires [22] that each stack of D6-branes is related to the O6-planes by a rotation in SU(3): denoting by $\theta_{i}$ the angles the D6-brane forms with 
TABLE I. D6-brane configuration for the three-family model.

\begin{tabular}{ccc}
\hline \hline Type & $N_{a}$ & $\left(n_{a}^{1}, m_{a}^{1}\right) \times\left(n_{a}^{2}, m_{a}^{2}\right) \times\left(n_{a}^{3}, \tilde{m}_{a}^{3}\right)$ \\
\hline$A_{1}$ & 8 & $(0,1) \times(0,-1) \times(2, \tilde{0})$ \\
$A_{2}$ & 2 & $(1,0) \times(1,0) \times(2, \tilde{0})$ \\
$B_{1}$ & 4 & $(1,0) \times(1,-1) \times(1, \widetilde{3 / 2})$ \\
$B_{2}$ & 2 & $(1,0) \times(0,1) \times(0, \widetilde{-1})$ \\
$C_{1}$ & $6+2$ & $(1,-1) \times(1,0) \times(1, \widetilde{1 / 2})$ \\
$C_{2}$ & 4 & $(0,1) \times(1,0) \times(0, \widetilde{-1})$ \\
\hline \hline
\end{tabular}

the horizontal direction in the $i$ th two-torus, supersymmetry preserving configurations must satisfy $\theta_{1}+\theta_{2}+$ $\theta_{3}=0$. In order to simplify the supersymmetry conditions within our search for realistic models, we will consider a particular ansatz: $\left(\theta_{1}, \theta_{2}, 0\right),\left(\theta_{1}, 0, \theta_{3}\right)$, or $\left(0, \theta_{2}, \theta_{3}\right)$.

Because of the smaller number of O6-planes in tilted configurations, the RR tadpole conditions are very stringent for more than one tilted torus. Focusing on tilting just the third torus, the search for theories with U(3) and $\mathrm{U}(2)$ gauge factors carried by branes at angles and three left-handed quarks turns out to be very constraining, at least within our ansatz. We have found essentially a unique solution. The D6-brane configuration with wrapping numbers $\left(n_{a}^{i}, \tilde{m}_{a}^{i}\right)$ is given in Table I.

The eight D6-branes labeled $C_{1}$ are split in two parallel but not overlapping stacks of six and two branes, and hence lead to a gauge group $\mathrm{U}(3) \times \mathrm{U}(1)$. Interestingly, a linear combination of the two U(1)'s is actually a generator within the SU(4) arising for coincident branes. This ensures that this $U(1)$ is automatically nonanomalous and massless (free of linear couplings to untwisted moduli) $[27,28]$, and turns out to be crucial in the appearance of hypercharge in this model.
For convenience we consider the eight D6-branes labeled $A_{1}$ to be away from the O6-planes in all three complex planes. This leads to two D6-branes that can move independently [hence give rise to a group $\mathrm{U}(1)^{2}$ ], plus their $\theta, \omega$, and $\Omega R$ images. These U(1)'s are also automatically nonanomalous and massless. In the effective theory, this corresponds to Higgsing of $\mathrm{USp}(8)$ down to $\mathrm{U}(1)^{2}$.

The open string spectrum is tabulated in Table II. The generators $Q_{3}, Q_{1}$, and $Q_{2}$ refer to the $\mathrm{U}(1)$ factor within the corresponding $\mathrm{U}(n)$, while $Q_{8}, Q_{8}^{\prime}$ are the $\mathrm{U}(1)$ 's arising from the $\operatorname{USp}(8)$. The hypercharge is defined as

$$
Q_{Y}=\frac{1}{6} Q_{3}-\frac{1}{2} Q_{1}+\frac{1}{2}\left(Q_{8}+Q_{8}^{\prime}\right) .
$$

From the preceding comments, $Q_{Y}$ as defined guarantees that $\mathrm{U}(1)_{Y}$ is massless. The theory contains three standard model families, plus one exotic chiral (but anomaly free) set of fields, and multiplets with vectorlike quantum numbers under the SM gauge group.

Even though the model is an explicit string realization of the brane world scenario, the string scale is of the order of four-dimensional Planck scale because standard model gauge interactions are embedded in different D6-branes. The experimental bounds on the Kaluza-Klein replica of standard model gauge bosons imply that the internal dimensions cannot be large [26].

Quarks, leptons, and Higgs fields live at different intersections; hence the Yukawa couplings among the Higgs and two fermions arise from a string world sheet of area $A_{i j k}$ (measured in string units) stretching between the three intersections [27], $Y_{i j k} \sim \exp \left(-A_{i j k}\right)$. Note that one family of quarks and leptons do not have renormalizable couplings with the Higgs field, due to the uncanceled $Q_{2}$ charges, and the only chiral multiplets which carry opposite $Q_{2}$ charges are charged under the weak SU(2).

TABLE II. Chiral spectrum of the open string sector in the three-family model. The nonAbelian gauge group is $\mathrm{SU}(3) \times \mathrm{SU}(2) \times \mathrm{USp}(2) \times \mathrm{USp}(2) \times \mathrm{USp}(4)$. Notice that we have not included the $a a$ sector piece, even though it is generically present in the model. The nonchiral pieces in the $a b, a b^{\prime}$, and $a a^{\prime}$ sectors are not present for branes at generic locations; hence they are not listed here.

\begin{tabular}{lrrrrrrr}
\hline \hline Sector & Non-Abelian Reps. & $Q_{3}$ & $Q_{1}$ & $Q_{2}$ & $Q_{8}$ & $Q_{8}^{\prime}$ & $Q_{Y}$ \\
\hline$A_{1} B_{1}$ & $3 \times 2 \times(1, \overline{2}, 1,1,1)$ & 0 & 0 & -1 & \pm 1 & 0 & $\pm \frac{1}{2}$ \\
& $3 \times 2 \times(1, \overline{2}, 1,1,1)$ & 0 & 0 & -1 & 0 & \pm 1 & $\pm \frac{1}{2}$ \\
$A_{1} C_{1}$ & $2 \times(\overline{3}, 1,1,1,1)$ & -1 & 0 & 0 & \pm 1 & 0 & $\frac{1}{3},-\frac{2}{3}$ \\
& $2 \times(\overline{3}, 1,1,1,1)$ & -1 & 0 & 0 & 0 & \pm 1 & $\frac{1}{3},-\frac{2}{3}$ \\
& $2 \times(1,1,1,1,1)$ & 0 & -1 & 0 & \pm 1 & 0 & 1,0 \\
$B_{1} C_{1}$ & $2 \times(1,1,1,1,1)$ & 0 & -1 & 0 & 0 & \pm 1 & 1,0 \\
& $(3, \overline{2}, 1,1,1)$ & 1 & 0 & -1 & 0 & 0 & $\frac{1}{6}$ \\
$B_{1} C_{2}$ & $(1, \overline{2}, 1,1,1)$ & 0 & 1 & -1 & 0 & 0 & $-\frac{1}{2}$ \\
$B_{2} C_{1}$ & $(1,2,1,1,4)$ & 0 & 0 & 1 & 0 & 0 & 0 \\
& $(3,1,2,1,1)$ & 1 & 0 & 0 & 0 & 0 & $\frac{1}{6}$ \\
$B_{1} C_{1}^{\prime}$ & $(1,1,2,1,1)$ & 0 & 1 & 0 & 0 & 0 & $-\frac{1}{2}$ \\
& $2 \times(3,2,1,1,1)$ & 1 & 0 & 1 & 0 & 0 & $\frac{1}{6}$ \\
$B_{1} B_{1}^{\prime}$ & $2 \times(1,2,1,1,1)$ & 0 & 1 & 1 & 0 & 0 & $-\frac{1}{2}$ \\
& $2 \times(1,1,1,1,1)$ & 0 & 0 & -2 & 0 & 0 & 0 \\
\hline \hline
\end{tabular}


This model is supersymmetric for some choice of complex structure moduli, which determine the angles satisfying the supersymmetry condition. The supersymmetry breaking effect when the condition is violated is reflected as a Fayet-Iliopoulos term for the U(1) gauge fields. It is proportional to the deviation from the supersymmetric limit, and reproduces correctly the tachyonic scalar masses, and zero fermion masses. The corresponding $D$ term is expected to be canceled by vacuum expectation values of the tachyonic scalar fields, hence shifting the configuration to a corrected vacuum, where some intersecting D6-branes are recombined, triggering gauge symmetry breaking.

It is quite remarkable that these chiral models are related to the nonchiral model of [4], by recombinations of the 3-cycles on which the D6-branes wrap. This is the $T$-dual of a $D=4$ version of the small instanton transition [32]. Clearly, our search is in no sense exhaustive. There exist different variants, in the framework described here, obtained by, e.g., (i) changing the additional branes not directly involved in the SM structure, (ii) allowing branes rotated at an angle in all three tori, and (iii) compactifying type IIA orientifold on a different orbifold which preserves $N=1$ supersymmetry. However, within our ansatz for the angles in the case of $\mathbf{Z}_{2} \times \mathbf{Z}_{2}$ orbifold, the requirements of supersymmetry, standard model-like gauge group and number of chiral families are rather stringent, and the model presented here is relatively unique. Let us note that D6-branes wrapping around supersymmetric 3 -cycles with three nontrivial angles contribute to some (but not all) tadpole conditions with the same sign as that of an O6-plane-a feature which is absent when all the D6-branes are parallel to some O6-planes. It would be interesting to explore such variants to eliminate the additional vectorlike matter and the extra exotics. We leave this for further investigation.

We thank Gerardo Aldazabal, Savas Dimopoulos, Jens Erler, Jaume Gomis, Luis Ibáñez, Paul Langacker, Raul Rabadán, and Matt Strassler for discussions. M.C. and G. S. thank the Theory Division at CERN for hospitality during the course of the work. A. M. U. thanks M. González for kind encouragement and support. This work was supported in part by U.S. Department of Energy Grant No. DOE-EY-76-02-3071 (M. C.), by the Class of 1965 Endowed Term Chair (M. C.), UPenn SAS Dean's funds (G. S.), and the NATO Linkage Grant No. 97061 (M. C.).

[1] For reviews, see, e.g., B. R. Greene, in Lectures at Trieste Summer School on High Energy Physics and Cosmology (World Scientific, Singapore, 1990); F. Quevedo, hep-th/ 9603074; A. E. Faraggi, hep-ph/9707311; Z. Kakushadze, G. Shiu, S. H. Tye, and Y. Vtorov-Karevsky, Int. J. Mod. Phys. A 13, 2551 (1998); G. Cleaver, M. Cvetič, J. R. Espinosa, L. L. Everett, P. Langacker, and J. Wang, Phys. Rev. D 59, 055005 (1999), and references therein.

[2] B. A. Ovrut, hep-th/9905115, and references therein.

[3] C. Angelantonj, M. Bianchi, G. Pradisi, A. Sagnotti, and Ya. S. Stanev, Phys. Lett. B 385, 96 (1996).
[4] M. Berkooz and R. G. Leigh, Nucl. Phys. B483, 187 (1997).

[5] Z. Kakushadze and G. Shiu, Phys. Rev. D 56, 3686 (1997); Nucl. Phys. B520, 75 (1998); Z. Kakushadze, Nucl. Phys. B512, 221 (1998).

[6] G. Zwart, Nucl. Phys. B526, 378 (1998); D. O’Driscoll, hep-th/9801114.

[7] G. Shiu and S.-H. H. Tye, Phys. Rev. D, 58, 106007 (1998).

[8] G. Aldazabal, A. Font, L. E. Ibáñez, and G. Violero, Nucl. Phys. B536, 29 (1999).

[9] Z. Kakushadze, Phys. Lett. B 434, 269 (1998); Phys. Rev. D 58, 101901 (1998); Nucl. Phys. B535, 311 (1998).

[10] M. Cvetič, M. Plümacher, and J. Wang, J. High Energy Phys. 0004, 004 (2000).

[11] M. Klein and R. Rabadán, J. High Energy Phys. 0010, 049 (2000).

[12] M. Cvetič, A. M. Uranga, and J. Wang, Nucl. Phys. B595, 63 (2001).

[13] M. Cvetič, L. Everett, P. Langacker, and J. Wang, J. High Energy Phys. 9904, 020 (1999).

[14] J. Park, R. Rabadán, and A. M. Uranga, Nucl. Phys. B570, 38 (2000).

[15] M. Cvetič and P. Langacker, Nucl. Phys. B586, 287 (2000).

[16] M. Bianchi, G. Pradisi, and A. Sagnotti, Nucl. Phys. B376, 365 (1992); M. Bianchi, Nucl. Phys. B528, 73 (1998); E. Witten, J. High Energy Phys. 9802, 006 (1998); Z. Kakushadze, G. Shiu, and S.-H.H. Tye, Phys. Rev. D 58, 086001 (1998).

[17] C. Bachas, hep-th/9503030.

[18] C. Angelantonj, I. Antoniadis, E. Dudas, and A. Sagnotti, Phys. Lett. B 489, 223 (2000).

[19] R. Blumenhagen, B. Körs, and D. Lüst, J. High Energy Phys. 0102, 030 (2001).

[20] R. Blumenhagen, L. Görlich, and B. Körs, Nucl. Phys. B569, 209 (2000); J. High Energy Phys. 0001, 040 (2000).

[21] S. Förste, G. Honecker, and R. Schreyer, Nucl. Phys. B593, 127 (2001); J. High Energy Phys. 0106, 004 (2001).

[22] M. Berkooz, M. R. Douglas, and R. G. Leigh, Nucl. Phys. B480, 265 (1996).

[23] I. Antoniadis, E. Dudas, and A. Sagnotti, Phys. Lett. B 464, 38 (1999); G. Aldazabal and A. M. Uranga, J. High Energy Phys. 9910, 024 (1999).

[24] G. Aldazabal, L. E. Ibáñez, and F. Quevedo, J. High Energy Phys. 0001, 031 (2000); 0002, 015 (2000).

[25] G. Aldazabal, L. E. Ibanez, F. Quevedo, and A. M. Uranga, hep-th/0005067.

[26] R. Blumenhagen, L. Görlich, B. Körs, and D. Lüst, J. High Energy Phys. 0010, 006 (2000).

[27] G. Aldazabal, S. Franco, L. E. Ibáñez, R. Rabadán, and A. M. Uranga, hep-th/0011073; J. High Energy Phys. 0102, 047 (2001).

[28] L. E. Ibáñez, F. Marchesano, and R. Rabadán, hep-th/ 0105155.

[29] E. Dudas and J. Mourad, Phys. Lett. B 486, 172 (2000); R. Blumenhagen and A. Font, Nucl. Phys. B599, 241 (2001).

[30] M. Cvetič, G. Shiu, and A. M. Uranga, Report No. UPR943-T, CERN-TH/2001-182.

[31] C. Angelantonj and R. Blumenhagen, Phys. Lett. B 473, 86 (2000).

[32] E. Witten, Nucl. Phys. B460, 541 (1996). 